\title{
Réécriture et recomposition dans le manuscrit du Fou d'Elsa
}

Les « Apocryphes des derniers jours »

\section{Suzanne Ravis-Françon}

\section{(2) OpenEdition}

\section{Journals}

Édition électronique

URL : https://journals.openedition.org/genesis/5208

DOI : 10.4000/genesis.5208

ISSN : 2268-1590

\section{Éditeur :}

Presses universitaires de Paris Sorbonne (PUPS), Société internationale de génétique artistique littéraire et scientifique (SIGALES)

\section{Édition imprimée}

Date de publication : 15 juillet 2020

Pagination : 55-67

ISBN : 979-10-231-0679-4

ISSN : 1167-5101

\section{Référence électronique}

Suzanne Ravis-Françon, "Réécriture et recomposition dans le manuscrit du Fou d'Elsa ", Genesis [En ligne], 50 | 2020, mis en ligne le 15 juillet 2021, consulté le 12 janvier 2023. URL : http:// journals.openedition.org/genesis/5208; DOI : https://doi.org/10.4000/genesis.5208 


\title{
Réécriture et recomposition dans le manuscrit du Fou d'Elsa Les «Apocryphes des derniers jours"
}

\author{
Suzanne Ravis-Françon
}

$\mathrm{E}$ uvre singulière, longuement mûrie, à la fois poème et roman, Le Fou d'Elsa est publié en 1963, dans une période de sa vie où Aragon s'interroge de plus en plus sur le temps, celui d'une vie d'homme comme celui de l'Histoire; autant d'ouvertures sur ces civilisations que l'Occident dans son entreprise de colonisation a méprisées ${ }^{1}$. En premier lieu, les cultures de l'Islam, qu'il a déjà approchées en s'intéressant aux littératures du monde soviétique $^{2}$. Le siège et la prise de Grenade constituent le cadre historique du Fou d'Elsa. Ces événements dont Aragon esquisse l'histoire, considérée du point de vue des vaincus, signent la fin de la coexistence, fragile mais réelle, entre les trois religions et cultures du Livre.

En miroir de ce bouleversement, Aragon place à Grenade la figure du Medjnoûn, réincarnation des poètes mystiques persans. Ce personnage est amoureux d'une femme, Elsa, qui vivra au XXe siècle et qu'il ne peut atteindre que dans son chant; ce chant, que reprendront au fil des siècles d'autres poètes ou mystiques, annonce l'avènement des «temps du couple».

Au cœur du Fou d'Elsa, le titre «Apocryphes des derniers jours » et ses trois poèmes occupent une place singulière, en ce sens qu'Aragon y reprend d'une façon plus personnelle et intime le problème de la vie et de la mort qui court tout au long de l'œuvre.

\section{L'état du manuscrit}

Quel chercheur travaillant sur Le Fou d'Elsa ne souhaiterait pas s'engager «sur la piste des secrets d'une création ${ }^{3}$ »? Le manuscrit du Fou d'Elsa ${ }^{4}$ se prête pourtant malaisément à l'analyse génétique; il est en effet arrivé au fonds, en décembre 1982, «en vrac», selon l'expression de Renate Lance-Otterbein au début du catalogue du fonds :
Sur l'état du manuscrit [...], il n'existe aucune description. Jean Ristat fait travailler deux personnes à la mise en ordre des papiers. Du résultat de leur travail témoignent quelques photos en grands plans. À ce moment la plupart des documents étaient rangés en dossiers suspendus. Ces premières interventions étaient repérables par l'écriture au stylo feutre sur des chemises vertes et un regroupement de correspondances par thèmes. Les rudiments d'inventaire que j'ai pu établir à partir du printemps 1983 partirent de cette base. Le fonds possédait à cette époque une estampille ovale en caoutchouc «CNRS Paris » dont on marquait les manuscrits à l'encre rouge [...]. L'autre mesure apportée très tôt était la numérotation des feuillets à l'aide d'un folioteur mécanographique mais cette consigne fut abandonnée lorsqu'on s'est rendu compte que la foliotation impliquait la mise en ordre et le tri préalable des dossiers parvenus en vrac.

Trente ans plus tard, dans sa «Note sur le texte», Olivier Barbarant souligne que «cet abondant matériel, sans doute le plus volumineux dont nous disposions, fait cependant problème en raison de l'absence de classement du dossier remis au fonds dans un incontestable désordre ${ }^{5}$ ». Un patient travail permet cependant, dans de nombreux cas, de distinguer les étapes successives de la recherche malgré l'ampleur

1. Les deux décennies qui précèdent la publication du Fou d'Elsa ont vu la disparition des empires coloniaux. L'Algérie vient d'accéder à l'indépendance.

2. Louis Aragon, Littératures soviétiques, Paris, Denoël, 1955.

3. Louis Aragon, «D'un grand art nouveau : la recherche», Essais de critique génétique, $\mathrm{n}^{\circ} 13$, Textes et manuscrits, série publiée par Louis Hay, 1979. Discours d'Aragon prononcé lors de la remise de son legs au CNRS le 4 mai 1977.

4. Le dossier se compose de 798 folios manuscrits (brouillons, manuscrits de travail, mises au net), 1816 dactylogrammes ou «tapuscrits », et des épreuves.

5. Olivier Barbarant, «Note sur le texte», dans Louis Aragon, Euvres poétiques complètes $(O P C)$, t. II, Paris, Gallimard, coll. «Bibliothèque de la Pléiade», 2007, p. 1546. 
inhabituelle du dossier. C'est le cas en particulier pour les trois derniers poèmes du dossier génétique.

\section{Les Apocryphes des derniers jours}

La réunion des trois poèmes subsumés sous le surtitre unique d' «Apocryphes» donne de l'ampleur à la phase ultime du Fou d'Elsa, mais soulève d'autant plus de questions que l'interprétation de leur coprésence est loin d'être évidente. Les trois éléments du triptyque sont-ils complémentaires, contradictoires ou sans relation? La situation de chacun dans la succession forcément linéaire de la section finale, et leur place définitive, a fait l'objet, au niveau du manuscrit, de décisions importantes non commentées par l'auteur.

Cet ensemble très structuré offre au lecteur une numérotation stricte :

I. «... Et si beau que me fût le jour»

II. «Le vrai zadjal d'en mourir»

III. «Ô impie»

L'ordre dans lequel Aragon a placé ces trois textes mérite attention. À ce moment décisif de la réalisation où l'œuvre écrite part pour l'impression, le poème «Et si beau que me fût le jour» quitte la partie «Les chants du Medjnoûn» pour venir se placer au début des «Apocryphes»; le surtitre «Apocryphes...» apparaît en ajout manuscrit sur le tapuscrit; le titre «Ô impie» apparaît également en ajout manuscrit; enfin le surprenant «zadjal», entièrement manuscrit, est placé entre les poèmes dactylographiés I et III. Sa foliotation 676 et 677, écrite à la main par Aragon, ne voisine pas avec d'autres chiffres barrés, comme c'est le cas pour les deux autres poèmes. Ce détail laisse penser que «Le vrai zadjal d'en mourir» a été placé, ou même rédigé, au moment où Aragon a fixé définitivement l'ordre des poèmes. L'ordre, ou plutôt ce que Jean Ristat, répondant à la question de Renate Lance, appelle l' «ordonnancement» : une phase seconde de l'écriture, où se mettent en place la disposition des textes, souvent leurs titres, et les décisions majeures de composition. «Aragon, dit-il, me parlait du classement. Comme il me parlait de battre les cartes. Comme il me parlait sans arrêt de l'ordre.» Une œuvre était «achevée» à ses yeux, pense Jean Ristat, «lorsque le classement [était] trouvé, lorsque l'ordre du manuscrit, la mise en espace, [était] juste ${ }^{6}{ }_{»}$.

Ces poèmes sont consacrés à trois thèmes fondamentaux : la mort, l'amour et la question de l'existence ou de la non-existence de Dieu, et partant, de l'absence d'immortalité. Mais ces trois pôles essentiels de la méditation ne doivent pas être pris isolément : la mort les habite tous les trois ; et ce sont les différentes réponses du poète à la vision et à la pensée de la mort imminente, qui composent une sorte de débat polyphonique.

Dans «... Et si beau que me fût le jour», l'amour charnel s'allie à la conception de la vie assimilée à une fiévreuse course à la mort; à travers les battements du cœur, ce tambour de chair et de sang, le poème évoque cette lutte incessante «en nous» de la vie et de la mort, dans un mouvement vertigineux des contraires qui cherche sa résolution :

Ô vie ô mort double déni que se fasse en nous l'harmonie.

«Le vrai zadjal d'en mourir» apporte-t-il une réponse à cette aspiration ? Il semble opposer à l'angoisse de la décomposition le dépassement vers l'amour absolu, la sérénité dans l'oblation à l'aimée. Il dit le bonheur de celui que son amour consume jusqu'à en mourir. On imagine difficilement qu'un tel poème eût trouvé place avant «...Et si beau que me fût le jour »; le choix d'un tel ordre aurait signé la négation de la conception plus éthérée de l'amour exaltée par le zadjal.

Le troisième poème dans l'édition définitive, «Ô impie», déplace le face à face avec la mort vers la question de Dieu. L'amour n'est pas évoqué directement, mais à travers la mémoire de «tout ce que j'ai vu tout ce que j'ai aimé». Le poème prolonge «Le vrai zadjal d'en mourir», où le poète se disait «sourd / Au chant s'il n'est de son amour» et «Aveugle au jour d'après son jour / Ses yeux sur toi seule fermés». Mais c'est la négation de toute transcendance, l'affirmation de l'immuabilité de la nature. Le problème de l'existence de Dieu est posé à maintes reprises dans Le Fou d'Elsa ${ }^{7}$ et notamment dans «Les Falâssifa», où Aragon reprend les débats des théologiens de l'Islam sur le Bien et le Mal et sur la part que Dieu y prend. Les deux poèmes «Blasphème pour être mis à mort» et «Dieu est

6. Jean Ristat et Renate Lance-Otterbein, «Comment Aragon écrivaitil ?», Recherches croisées Aragon-Elsa Triolet (RCAET), $\mathrm{n}^{\circ} 1$, Besançon, Les Annales Littéraires de l'Université de Franche-Comté, 1988, p. 16. 7. Wolfgang Babilas en a fait une recension et une analyse complètes : «Dieu dans Le Fou d'Elsa», Études sur Louis Aragon, II, Münster, Nodus-Publikationen, 2002, p. 685-719; et dans les actes du colloque Le Rêve de Grenade, Publications de l'Université de Provence, 1996, p. 302-312. 


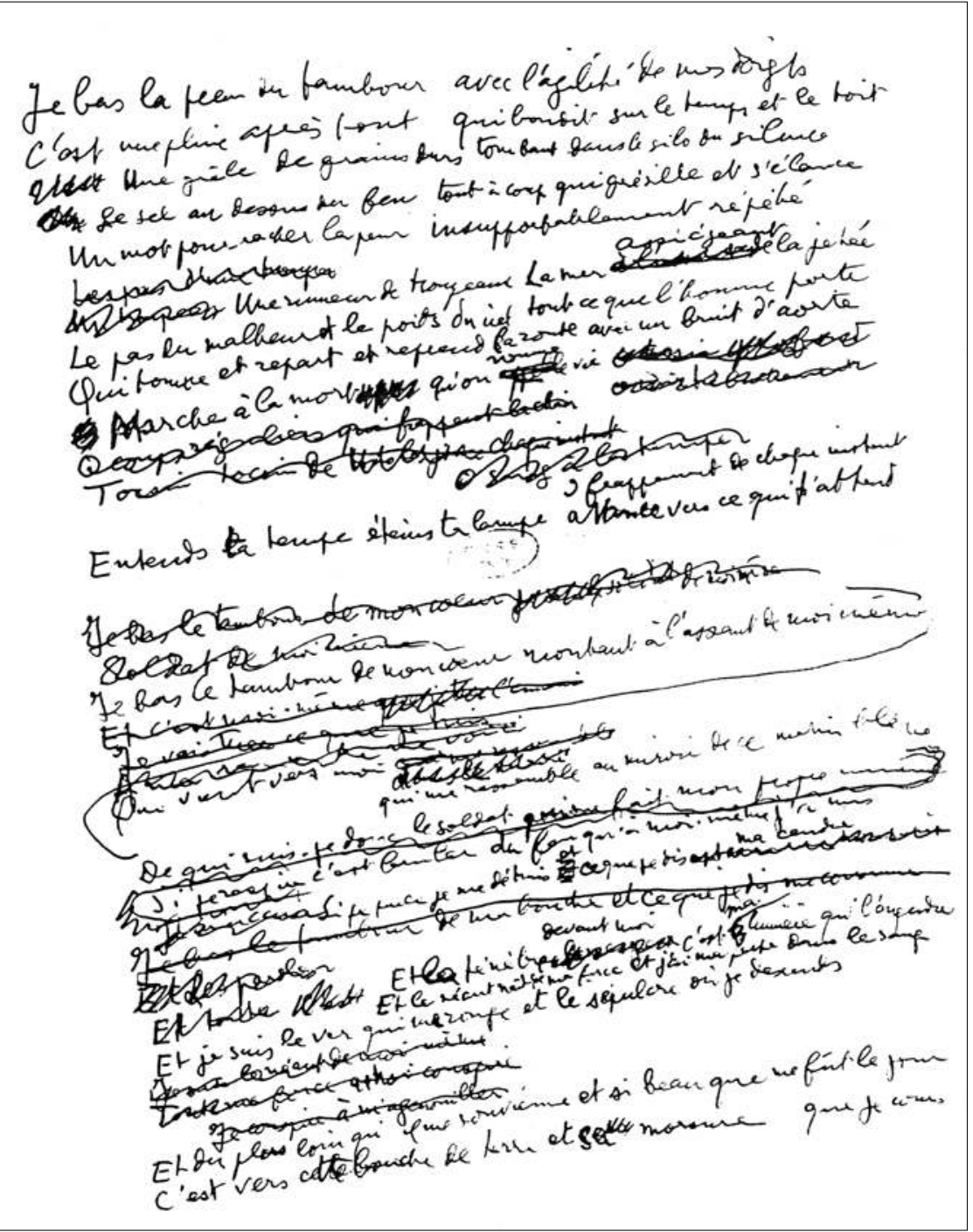

Fig. 1 : Premier feuillet de «Je bas la peau du tambour» (dossier «Annexes»).

(C) Paris, BnF, avec l'aimable autorisation de Jean Ristat

dans celui qui le nie» posent le problème de la révolte contre Dieu; le poème «Ô Impie» le résout en opposant à l'angoisse de la mort la sérénité qu'inspire au mourant «cette joie étrange que sans moi l'eau continue à couler dans le verger / Sans moi l'aube à venir s'accouder aux collines ». Et s'il commence par dire le défi de l'homme face à celui qui se présente comme son créateur, les derniers vers de l'œuvre donnent une double impression, d'apaisement et de victoire de l'homme à travers la «sourate imaginaire» finale. La place ultime de ces vers et leur ton peuvent laisser penser qu'ils expriment le message dernier de l'auteur.

\section{«Et si beau que me fût le jour»}

L'étude génétique ne s'applique avec profit qu'au premier des Apocryphes; pour «Le vrai zadjal» nous n' avons aucune variante; pour «Ô impie» nous disposons bien d'un manuscrit, mais il est peu différent de l'état définitif. Pour ces deux poèmes, notre analyse prendra donc une autre forme.

En ce qui concerne «Et si beau que me fût le jour», le dossier «Annexes ${ }^{8}$ » contient deux feuillets de brouillons manuscrits sans titre ni numéro, placés juste avant le dernier document du dossier (fig. 1). La lecture de ces «Annexes » est importante pour éclairer la genèse du poème. Les nombreux fragments écrits et non retenus laissent penser qu'on est ici en présence de la première ou de l'une des premières écritures du poème.

Dans la première strophe, «Je bas la peau du tambour» (corrigée plus tard par l'auteur «Je bats »), on voit qu'Aragon a essayé puis biffé quelques mots, comme «tocsin tocsin». Seul le vers «Une rumeur de troupeaux La mer assiégeant

8. Le dossier «Annexes» est constitué de 65 feuillets disparates, dont deux (sans titre) concernent les «Apocryphes des derniers jours ». On ignore si ces feuillets ont été rassemblés par Aragon lui-même. 
la jetée» sera ensuite retouché : «Une mer», pour ajouter la syllabe manquante 9 .

La deuxième strophe a connu plusieurs tentatives d'écriture; certains fragments n'ont pas été conservés, comme «Je vais tuer ce que je suis», «Je suis le néant de moimême», «Toute ma force en moi conspire», «Je conspire à m'agenouiller». Les trois premiers vers de la strophe sont écrits dans le désordre. La fin de la seconde strophe ne changera plus. La puissance des images, la force destructrice du poème, sont incommensurables.

La troisième strophe, en revanche, semble écrite d'un seul mouvement. Les dix vers de ce brouillon comptent douze fois le mot «tambour», comme un battement lancinant. Les deux derniers vers, malgré quelques ratures, peuvent être lus :

Sur l'infini des bras unis et désunis seule harmonie

Roule tambour comme un déni roule tambour ton insomnie.

Cette version du poème, nous allons la retrouver dans les dossiers 7A et 10DE.

Le dossier 7A qui contient, entre autres textes manuscrits, une partie des «Chants du Medjnoûn», nous apporte une indication importante. Nous y trouvons un manuscrit qui est la mise au propre du texte que nous venons de déchiffrer dans le dossier «Annexes». Ce texte est repris intégralement. Il porte, paginé de la main d'Aragon (au feutre orange), le numéro 74. Dans le même dossier nous trouvons un feuillet 73 paginé de la même façon, manuscrit du poème «Faudra-t-il que le chant finisse», qui prendra plus tard le titre «L'ENCORE». Or nous savons que ce poème est le dernier des «Chants du Medjnoûn». On peut donc raisonnablement faire l'hypothèse que le folio 74 («Et si beau que me fût le jour») était destiné à figurer en dernière place dans les «Chants du Medjnoûn».

Le dossier 10DE donne la même indication sur la place de ces deux poèmes que l'on retrouve ici sous la forme de trois tapuscrits et un manuscrit. Pour faciliter la lecture, nous leur donnerons un code. Le premier tapuscrit (T1) reprend le texte «Faudra-t-il que le chant finisse»; il est paginé 71 (à la machine). Le deuxième tapuscrit (T2) numéroté 72 à la machine reprend le texte «Et si beau que me fût le jour » tel qu'il était, manuscrit, dans le dossier 7A. Ce qui prouve qu'au moment où Aragon donnait à dactylographier les «Chants du Medjnoûn», ce poème y était encore inclus.
Le troisième tapuscrit (T3) (fig. 2) reprend «et si beau que me fût le jour» mais y apporte des corrections importantes : le mot «tambour» qui était écrit douze fois en T2 avec un effet de martèlement lancinant n'apparaît plus que trois fois, et sous la forme «altambour» qui non seulement tire le lexique vers la langue arabe mais accentue certaines sonorités vocaliques ${ }^{10}$. C'est surtout le ton et la portée de la troisième strophe qui sont nouveaux; la conjonction de la vie et de la mort y est affirmée non seulement dans l'invocation du septième vers mais aussi à travers le rapprochement cruel entre les lits et les charniers; l'invocation «Ô vie ô mort» se termine par le souhait que s'établisse entre ces contraires une harmonie. Notons que l'harmonie n'est plus celle, assez naturelle, «des bras unis et désunis » mais qu'elle est désormais attendue en chacun de nous entre la vie et la mort. Nous donnons dans leurs deux écritures successives quelques-uns des vers qui traduisent cette nouvelle orientation du poème vers une coloration plus grave (encadré 1).

Le même dossier 10DE contient aussi un manuscrit portant, de la main d'Aragon, l'indication «non numéroté » (fig. 3); il est visiblement postérieur au tapuscrit T3. L'auteur a-t-il craint que ce tapuscrit, remanié au stylo, ne soit pas suffisamment lisible pour la dactylo? En le recopiant à la main il a omis le $8 \mathrm{e}$ vers ( Qui tombe et repart et reprend la route avec un bruit d'aorte») et il a donné une copie fautive du $10^{\mathrm{e}}$ vers ( $«$ Entends ta tempe éteinte éteins ta lampe avance vers ce qui t'attend»). De ce fait, ce vers compte 18 syllabes au lieu de 16 .

C'est sur la base de ce texte manuscrit de 10DE que la dactylographe a travaillé pour aboutir aux tapuscrits que nous trouvons dans les dossiers $1 \mathrm{~B}$ et $2 \mathrm{C}$ qui seront repris dans toutes les versions imprimées!

Le dossier $3 \mathrm{E}$ contient un feuillet intitulé «A placer» (fig. 4), donnant les titres ou les premiers vers d'un ensemble de poèmes qui n'ont pas encore trouvé leur place mais qu'on peut supposer déjà écrits puisqu'Aragon en

9. Maryse Vassevière a attiré l'attention sur un article publié par Aragon dans France Nouvelle (5 novembre 1959) dans lequel on rencontrait déjà, pour parler du cœur battant, les images et le rythme retrouvés ici : «Aragon vous parle : étude d'une chronique», RCAET, ${ }^{\circ} 6,1998$, p. 175-206.

10. Avec cette substitution le premier vers passe de 16 à 17 syllabes. 


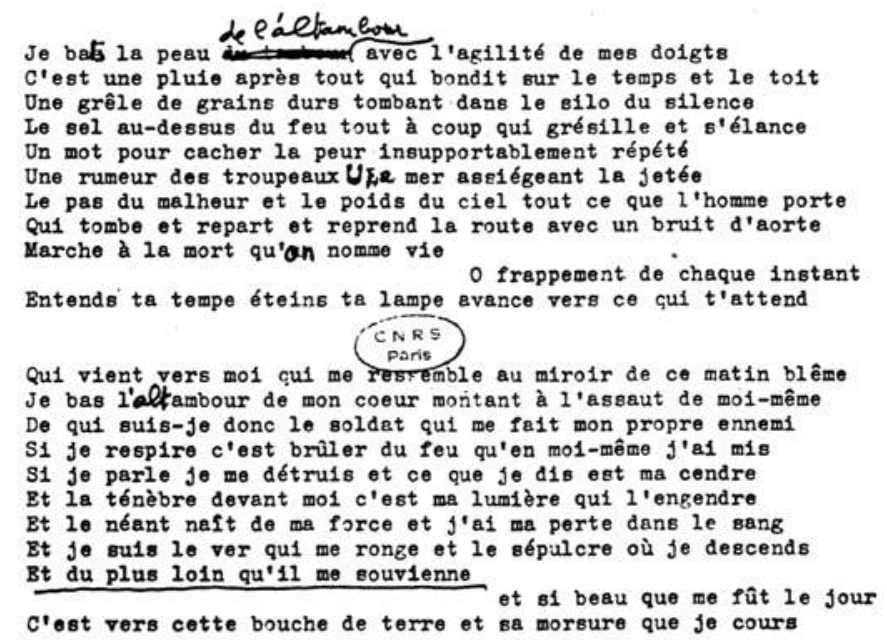

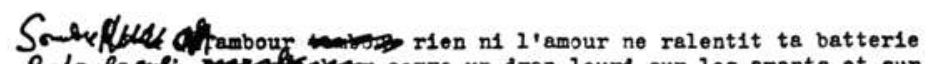

Retombe onf come un drap lourd fur les emants et sur leurs cris

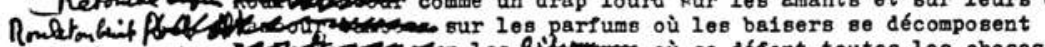

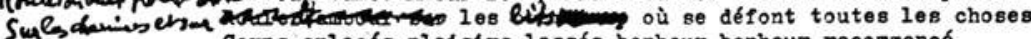

Corps enlacés pla1sirs lassés bonheur bonheur recomrencé
La longue étreinte comme un ch1ffre dans la chair aux yeux versés

La longue étreinte comme un chiffre dans la châr aux yeux versés

Prappe plus fort d'autant plus fort que c'est le dernier bal

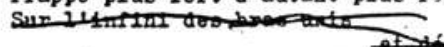

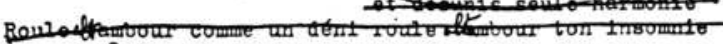

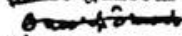

Hesprati

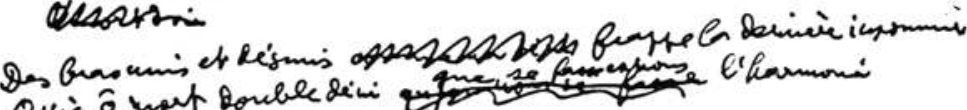

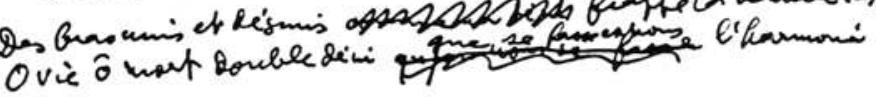

Fig. 2 : «Je bats la peau de l'altambour» (dossier 10DE).

() Paris, BnF, avec l'aimable autorisation de Jean Ristat

Roule tambour sur les charniers où se défont toutes les choses (première écriture) Sur les charniers et sur les lits où se défont toutes les choses (deuxième écriture)

Sur l'infini des bras unis et désunis seule harmonie (première écriture)

Des bras unis et désunis frappe la dernière insomnie (deuxième écriture)

Roule tambour comme un déni roule tambour ton insomnie (première écriture)

O vie ô mort double déni que se fasse en nous l'harmonie (deuxième écriture)*

(*) Nous indiquons en caractères gras les modifications apportées à la première écriture. 


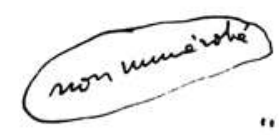

$$
\text { ‥ Et ribean peme fint le poin }
$$

Yebatsla pean de l'altambiour aval laglite'de mos soigh $C$ 'estune phie apistont quibondits sur Ce temps et le toit Une giele de graino durs tombant tan le jilo den silena Le selaudroon dn fen tout aiconp qrigisille de s'elance un wot pour cacher la peur insupportablenent reje'tré Une sumem des tronpears the mer asoiegeant ea jetei Le pas ou malhene of be poiss oncial tont ce quel'borme porte Marche à la novr quion wonume vie

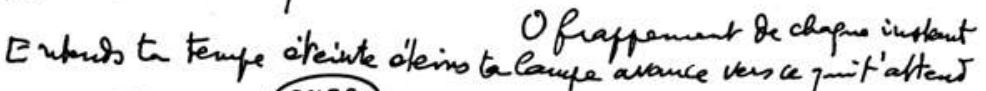

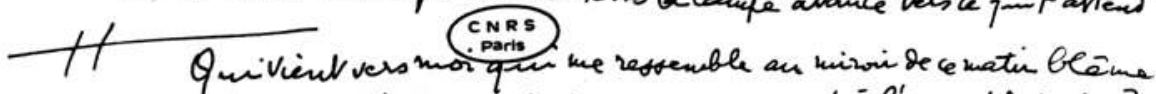

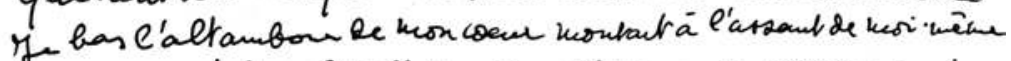

De quisur. ji donc be roldat quime fait mon prope ennemin

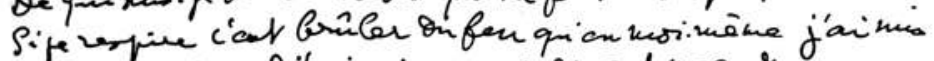
Sifie prle je meditinis elce que is dis eol ma cenre

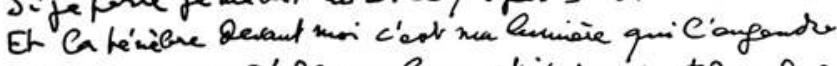

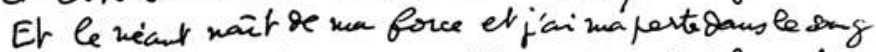

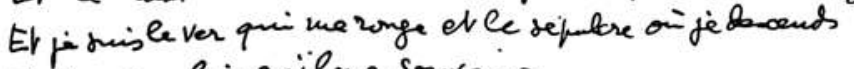
Erouplan boin quill me Sousame C'asl vass cette bouche de terre et sean queme fint le jour Sombre altambour sien mi l'anom ne zalentit tabaltaie

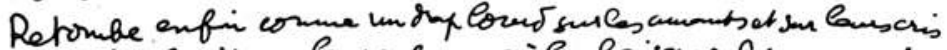
Roule ton exinit du les parfums ovices baitas $x$ décomproset

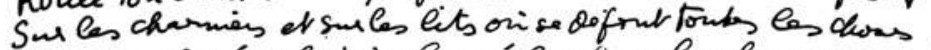

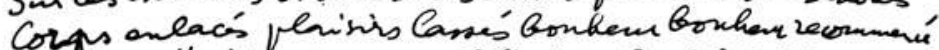

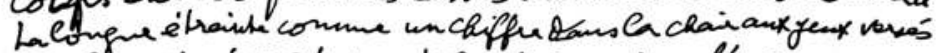

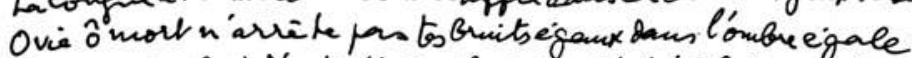
Finpe pens fort d'aulsut pens fort que c'ast ici le bermier bal Des brasmiset desunis frapte la dermicie inoommi.

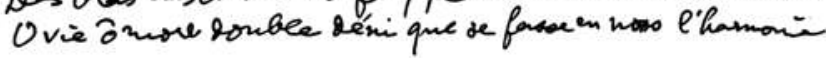

Fig. 3 : Manuscrit «non numéroté» «Et si beau que me fût le jour» (dossier 10DE).

(c) Paris, BnF, avec l'aimable autorisation de Jean Ristat 
RÉÉCRITURE ET RECOMPOSITION DANS LE MANUSCRIT DU FOU D'ELSA

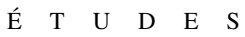

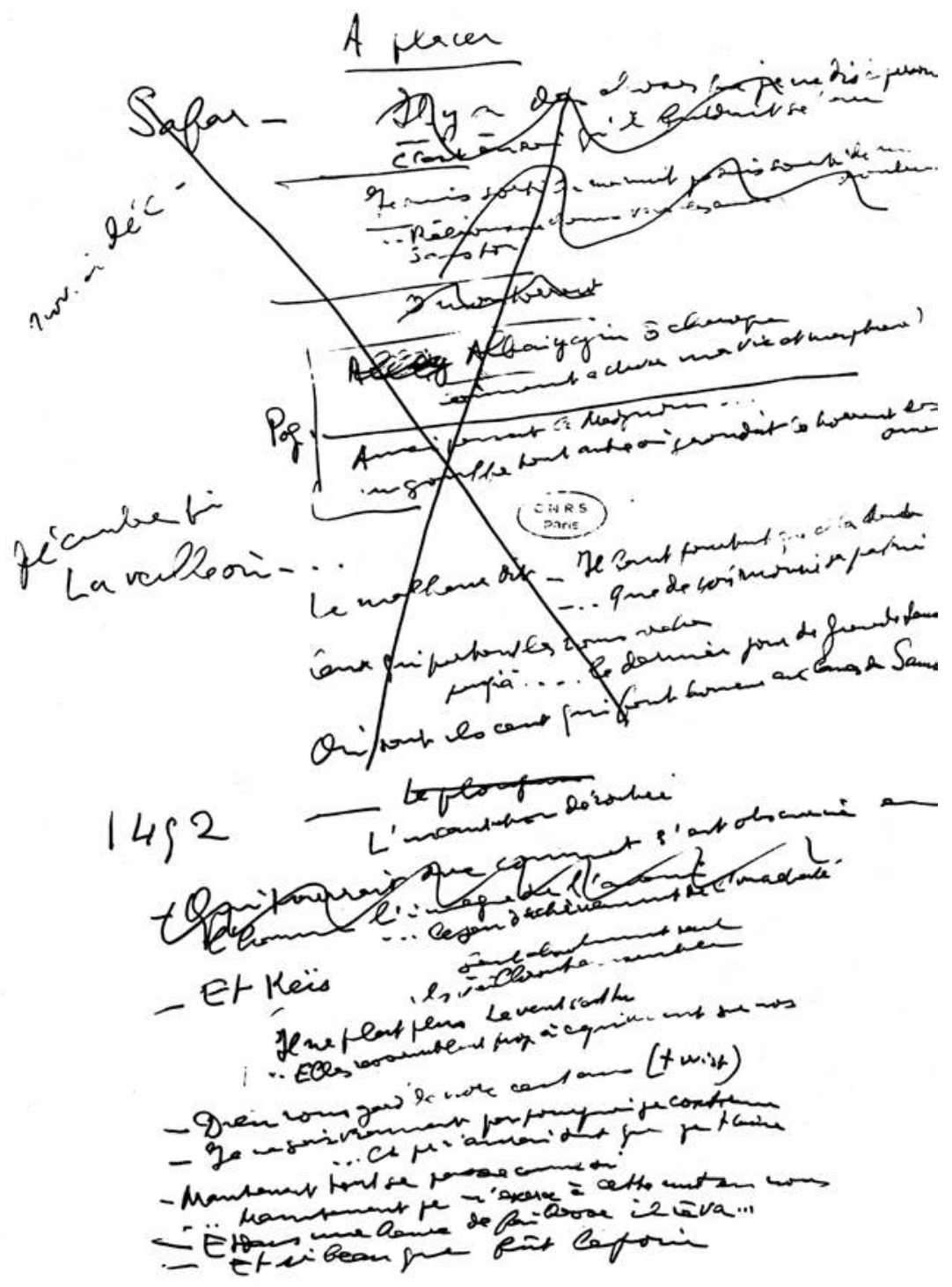

Fig. 4 : «A placer» (dossier $3 \mathrm{E})$.

(C) Paris, BnF, avec l'aimable autorisation de Jean Ristat

61 

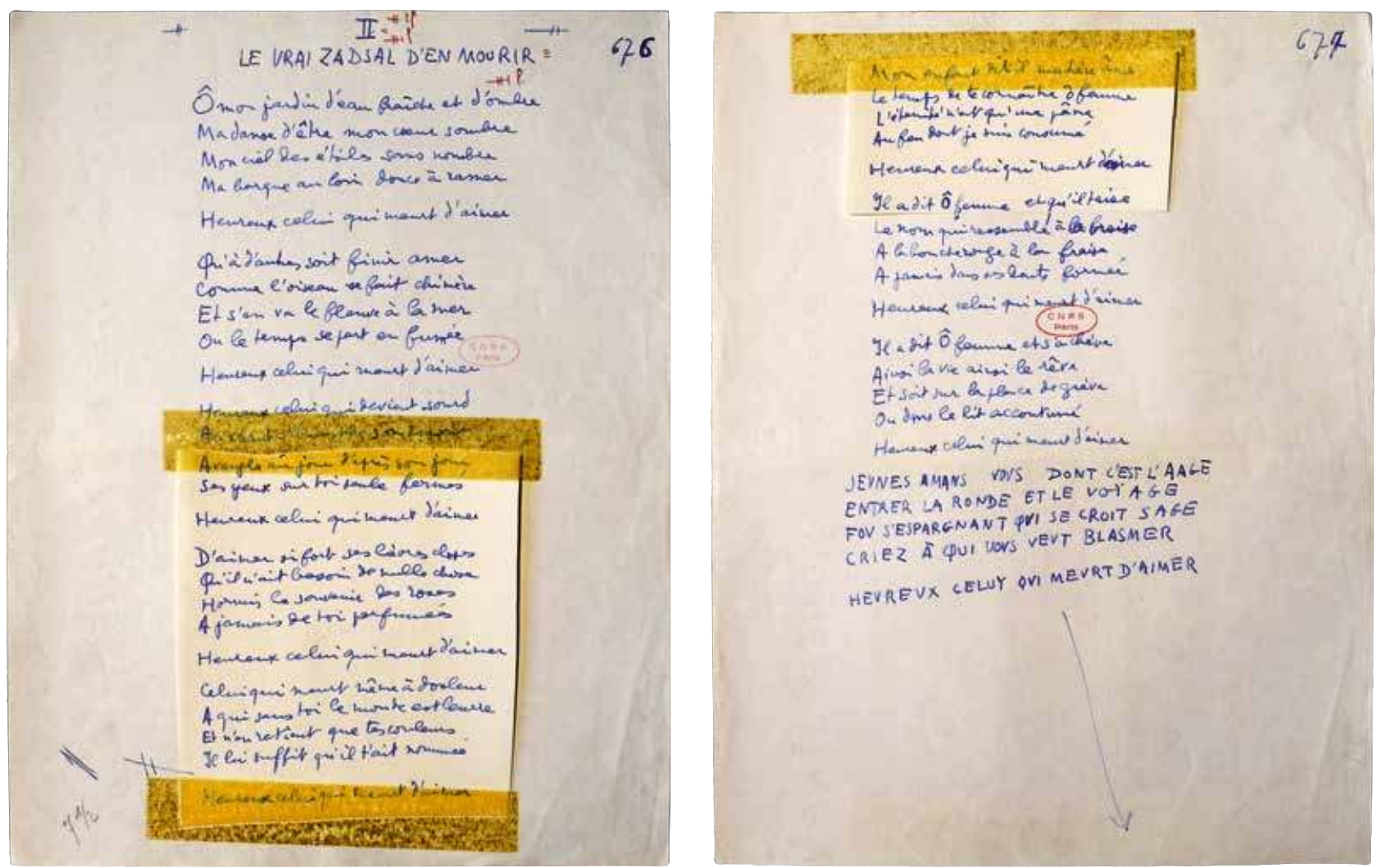

Fig. 5 et 6 : «Le vrai zadjal d'en mourir» (dossier 2 C).

(C) Paris, BnF, avec l'aimable autorisation de Jean Ristat

donne le premier vers. Dans la version définitive tous se situeront entre «Safar» et la fin du livre, autrement dit dans le troisième tiers de l'œuvre, ce qui nous permet de dater relativement ce document dans le mouvement de la création. Les deux derniers poèmes qui figurent tout au bas de cette liste sont «Et dans une heure de faiblesse il rêva» (qui est le début du poème «Ô Impie») et «Et si beau que fût le jour» (sans «me»). Notons qu'ils sont donnés dans l'ordre inverse de celui qu'ils suivront dans le manuscrit définitif.

Les dossiers $1 \mathrm{~B}$ et $2 \mathrm{C}$ contiennent des tapuscrits identiques qui sont la version définitive du texte, avec les erreurs que nous avons mentionnées, reprises dans toutes les éditions... Le dossier $2 \mathrm{C}$ donne un tapuscrit qui porte à la page 681 la griffe d'Aragon. C'est sous cette forme que le poème fut confié à l'imprimeur.

Dans ces dossiers apparaît l'ensemble des trois poèmes qui constituent les «Apocryphes des derniers jours » et leur ordonnancement interne. Le poème «... Et si beau que me fût le jour» est situé à la première place dans la triade. À la deuxième place nous trouvons un poème nouveau, dont il n'y a aucune trace dans les autres dossiers, «Le vrai zadjal d'en mourir ».

\section{«Le vrai zadjal d'en mourir»}

Ce poème chante un amour sublime, où «l'amour conduit à la perte de soi dans l'être aimé11» (fig. 5 et 6 ). Il est porté par neuf quatrains octosyllabiques soutenus par le refrain neuf fois répété, «Heureux celui qui meurt d'aimer». Cet élan passionné a, semble-t-il, été introduit entre les deux autres poèmes : «Le vrai zadjal d'en mourir» n'est pas mentionné dans le feuillet intitulé «A placer» (dossier $3 \mathrm{E})$, qui donnait à lire la longue liste de titres manuscrits terminée par les deux autres poèmes «apocryphes ». Aucune esquisse ne l'annonce dans les projets ou les versions intermédiaires de l'œuvre. Sa présence intégralement manuscrite placée dans le dossier dactylographié à remettre à l'imprimeur est un cas unique dans Le Fou d'Elsa.

Le document se présente sous la forme de deux manuscrits, constitués de papiers rassemblés, fixés par des fragments de scotch; la seule différence entre ces deux manuscrits tient au découpage de ces morceaux et donc à

11. Claire Kappler, Suzanne Thiolier-Méjean (éd.), Les Fous d'amour au Moyen Âge. Orient-Occident, Paris, L'Harmattan, 2007. 
la place des collages. Il n'y a pas une seule rature dans les deux textes que nous avons trouvés.

À défaut d'analyse génétique stricto sensu on peut s'interroger sur les origines intertextuelles de ce poème.

La plus grande partie du «Vrai zadjal d'en mourir» baigne dans une lumière qui vient de loin : de la poésie arabe et persane, celle de Djâmî, celle aussi de Nezâmi, ce poète soufi $\mathrm{du}$ XII ${ }^{\mathrm{e}}$ siècle qui, le premier, mit en vers la légende arabe de Layla et Medjnoûn, «l'histoire d'une passion amoureuse mutuelle qui ne s'accomplit que dans la mort ${ }^{12}$ ». Djâmî a repris sous le même nom l'histoire de Layla et Medjnoûn et c'est ce poème qu'Aragon a pu lire dans une traduction française du persan, par Chézy, publiée en 1807.

Il faut revenir à Nezâmi pour aller plus avant dans la compréhension de ce poème et de sa place au cœur des «Apocryphes ». De Nezâmi, qu'on range parmi les poètes soufis, Aragon nous dit que «son soufisme a le caractère des soufis de l'époque et il semble avoir été lié avec l'organisation des "akhi" (frères) qui constituaient des sortes de communes de travail13». Dans Littératures soviétiques, Aragon consacre une dizaine de pages à l'œuvre et à la pensée de Nezâmi «qui du XIIe siècle au XIII e fut le maître de tous les poètes non seulement de l'Azerbaïdjan mais des nations voisines, la Perse y comprise, et dont le "Khamsè", c'est-à-dire les cinq poèmes, fut plus imité que les poètes latins ou grecs ${ }^{14}$ ». De Nezâmi, Aragon a lu ce qu'il pouvait en lire en traduction russe, Le Trésor des secrets; il voit en lui «un athée, contempteur des dieux et des prophètes 15 ».

Si Aragon fait une telle place à Nezâmi c'est peut-être parce que cet exemple nourrit sa réflexion sur le rôle du poète et de la poésie. En octobre 1963, dans ses «Entretiens » avec Francis Crémieux qui l'interroge sur son rapport, lui athée, à la mystique de l'Islam, Aragon répond :

Un des plus grands mystiques arabes, Ibn Arabi, [a déclaré :] «Un être n'aime en réalité personne d'autre que son créateur.» [Mais] ne faudrait-il pas renverser la proposition et dire : «Qui j'aime me crée »? Ceci est la proposition mystique remise sur ses pieds. [...] La grandeur de la mystique, on la voit dans le fait que la poésie est tournée à Dieu. Moi, au contraire, je tourne la poésie à la femme, je tourne ma Kibla vers Elsa 16 .

En ayant à l'esprit ce texte d'Aragon, contemporain de la fin de l'écriture du Fou d'Elsa, on peut comprendre mieux en quoi ce zadjal peut être dit le vrai zadjal («vrai » parce qu'en exaltant la femme on donne à l'amour un horizon pleinement humain). Car le véritable objet d'amour, pour Aragon, n'est pas un Dieu illusoire. Il faut donc prendre à la lettre les images et les mots qui évoquent le paradis de la terre andalouse, ou arabe, ou persane : «le jardin d'eau fraîche et d'ombre», «le souvenir des roses », «Le nom qui ressemble à la braise / À la bouche rouge à la fraise... ». C'est bien d'amour qu'il s'agit, dans toutes ses formes humaines, qui n'appelle rien d'autre qu'une extension à toute l'humanité («Un jour pourtant un jour viendra couleur d'orange [...] Un jour d'épaule nue où les gens s'aimeront $17 »)$.

Comment comprendre le décalage de style qui s'instaure entre la dernière strophe et le reste du poème? L'écriture en majuscules fait penser à un texte inscrit dans le marbre ; le vocabulaire archaïsant («place de Grève», «meurt à douleur») évoque la poésie du Moyen Âge occidental et François Villon ${ }^{18}$. Jusque-là, la voix du poète a été celle d'un homme jeune comme l'était le Medjnoûn de Nezâmi et de Djâmî. Mais c'est Aragon maintenant qui parle, un homme qui se sent au seuil de la vieillesse, et c'est à des hommes jeunes, à ceux qui vont venir, qu'il passe le relais de son chant.

\section{«Ô Impie»}

Pour le poème «Ô Impie» (fig. 7 et 8 ), nous trouvons à la fin du dossier 10DE, immédiatement après les feuillets manuscrits et tapuscrits de «Et si beau que me fût le jour», quatre feuillets qui présentent deux versions manuscrites de ce poème :

12. Isabelle de Gastines, Layla et Majnûn, Paris, Fayard, 2017. Note liminaire, p. 10. C'est la première traduction en français du livre de Nezâmi.

13. Louis Aragon, Littératures soviétiques, op. cit., p. 188.

14. Ibid., p. 186.

15. Ibid., p. 189.

16. Louis Aragon, Entretiens avec Francis Crémieux, Paris, Gallimard, 1964 , p. 62-63.

17. Le Fou d'Elsa, édition originale, Paris, Gallimard, coll. «NRF», 1963, p. 377 (OPC II, p. 857).

18. Hervé Bismuth attire l'attention sur ces réminiscences dans son livre Le Fou d'Elsa d'Aragon, métissages linguistiques et discursifs, Dijon, Éditions universitaires de Dijon, 2007, p. 130. 

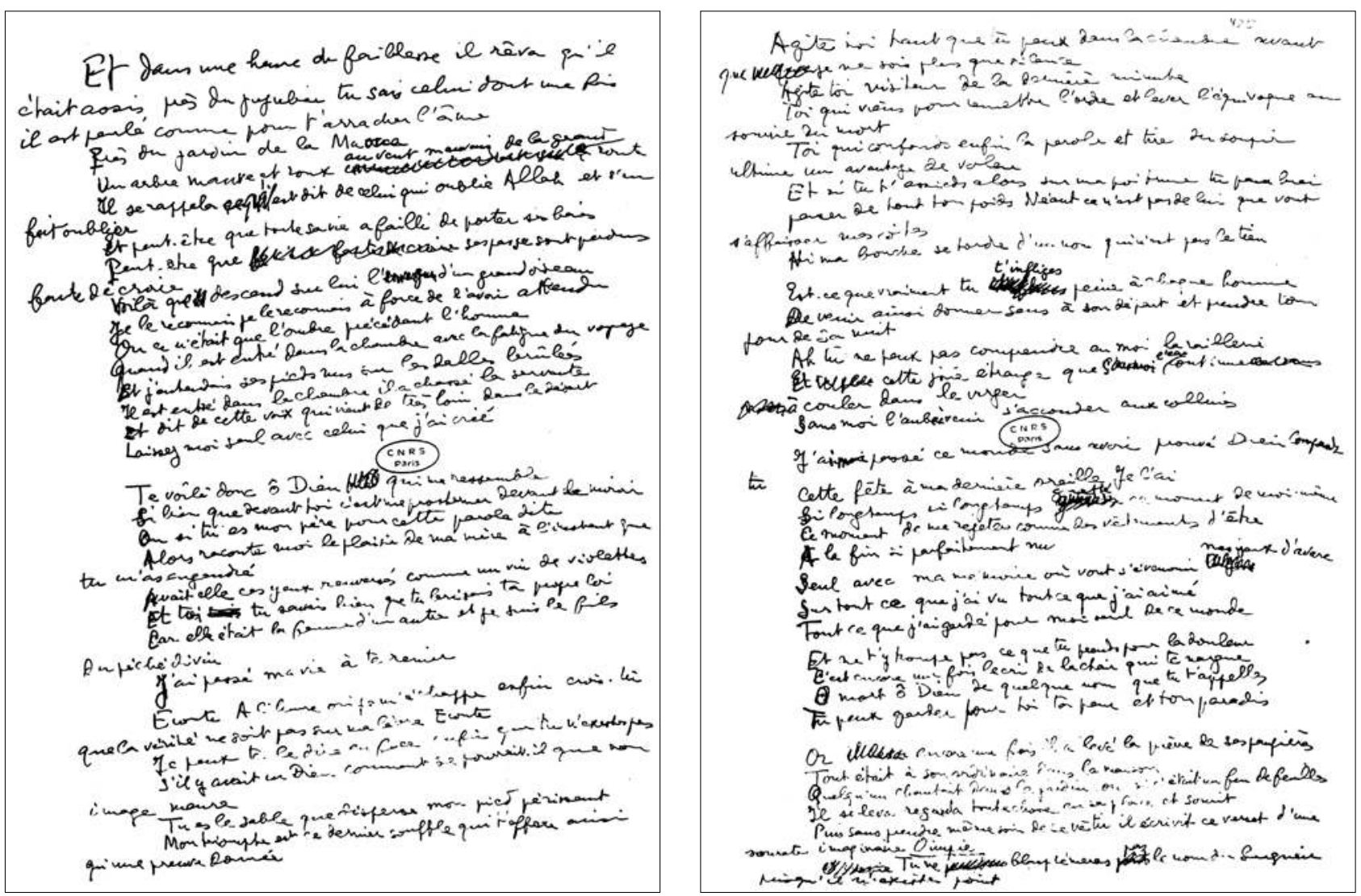

Fig. 7 et $8:$ «Et dans une heure de faiblesse...» (dossier 10DE).

(๑) Paris, BnF, avec l'aimable autorisation de Jean Ristat

- deux feuillets manuscrits sont une première version, sans titre, avec quelques mots biffés illisibles;

- un troisième feuillet portant la mention «non numéroté » est la mise au propre de la première page de cette première version;

- le quatrième feuillet ne porte que les deux derniers vers. Mais il faut ajouter à ces quatre feuillets un cinquième qui n'est pas dans le dossier 10DE; on le trouve en fac-similé dans le tome XIV de L'Euvre poétique ${ }^{19}$ : ce fac-similé appartient à la deuxième version du poème; il y manque les deux derniers vers; ce sont ceux que nous trouvons dans le quatrième feuillet du dossier 10DE.

Ces deux versions manuscrites ne portent pas de titre. Elles ne comportent que deux différences avec les tapuscrits que nous trouvons dans les dossiers $1 \mathrm{~B}$ et $2 \mathrm{C}$. Ces différences concernent la fin du poème. Dans les deux manuscrits on trouve «Il se leva regarda toute chose», tandis que les tapuscrits et le texte imprimé donnent «Il se leva regarder toute chose...», suggérant davantage une intention. Cet infinitif «regarder» n'apparaît nulle part dans les manuscrits ; il a donc été tapé par la dactylographe et validé par Aragon à la lecture du tapuscrit. La seconde différence concerne les deux dernières lignes : dans les manuscrits seuls les mots «Ô Impie» sont soulignés comme s'ils étaient destinés à devenir le titre de la sourate imaginaire; dans les tapuscrits c'est tout le verset qui est souligné comme si l'auteur avait voulu par ce soulignement marquer l'importance de ce «commandement» : «Ô Impie / Tu ne blasphémeras pas le nom du Seigneur puisqu'il n'existe point».

L'analyse génétique des manuscrits et tapuscrits du poème «Ô Impie » recueille une moisson assez mince si on s'en tient aux variantes. C'est dans l'intertextualité avec le Coran et avec d'autres textes de l'auteur, notamment, dans le Fou d'Elsa, le chapitre «Débat de l'avenir», que nous trouvons le plus de «grain à moudre».

$\mathrm{Si}$ «Le vrai zadjal d'en mourir» est tendu comme le mouvement d'une flèche porté par le souffle répété de son refrain, le poème «Ô Impie» est, lui, d'une grande complexité. Il s'ouvre par l'évocation d'un univers qu'on pourrait dire oriental et coranique : le lieu mythique appelé

19. Nous nous référons à l'édition dite «de luxe», en 15 volumes, du Livre Club Diderot, publiée en 1974 et 1981. Le fac-similé reproduit est face à la page 416. Apparemment ce feuillet n'a pas été restitué au fonds. 
par le Coran Sidrati-Montaha, le «jujubier de la dernière marche 20 » et le jardin de la Maroua sont empruntés à la sourate LIII («L'étoile»). L’homme affaibli qui craint d'avoir «failli» toute sa vie sans reconnaître Allah voit descendre sur lui le grand oiseau, «Simorgh 21 » ou «Ange fort et doué de sagacité» qui se tient au-dessus de lui 22 . Le dernier vers d'Aragon, à la fin de la première partie, «Laissez-moi seul avec celui que j'ai créé» (sans majuscule pour «je») s'inspire de la sourate LXXIV («Celui couvert d'un manteau »); «Laisse-moi seul avec celui que J'ai créé» correspond à la traduction du Coran de Régis Blachère. La majuscule « $\mathrm{J} »$ est ordinairement attribuée à la puissance divine d'un être surnaturel ; cette sourate met en garde celui qui s'est détourné de Dieu et a nié sa divinité, mais l'absence de majuscule (qu'elle soit volontaire ou non) donne à penser qu'ici c'est la figure du père d'Aragon qui surgit.

La seconde strophe voit l'affrontement du mourant avec celui qui se présente comme son créateur. Affrontement direct, brutal, blasphématoire. À qui s'adresse ce blasphème? Aragon reprend ici la posture blasphématoire du «Débat de l'avenir» qui opposait ceux pour qui l'avenir de l'homme est Dieu à ceux pour qui l'avenir de l'homme est l'homme ; ou, mieux encore, la femme. Ces textes fournissent un contrepoint à ce que nous dit «Ô Impie». Ici il n'est plus question de l'avenir mais simplement de «l'après » : après la mort, l'aube continuera de «venir s'accouder aux collines».

Tous les commentateurs ont vu, dans ce refus de la paternité symbolique, qu'on attribue à Dieu un glissement qui ouvre un espace d'ambiguïté, d'équivoque. Ce père qu'on défie, est-ce Dieu ou le père humain, le père d'Aragon? Et qui parle? Aragon philosophe mettant en question la conception patriarcale du Dieu des monothéismes, affirmant résolument son athéisme, ou Aragon le fils adultérin de Louis Andrieux reniant son père ? Sans doute les deux à la fois. Mais cela ne suffit peut-être pas.

Car le poème peut se lire à deux et même à trois niveaux différents : on peut y lire une déclaration d'athéisme radical et cette lecture est possible jusqu'à la fin du poème. Sans s'appesantir sur la figure du préfet de police de Paris, Louis Andrieux, aux antipodes des positions politiques d'Aragon, on peut y lire le cri de défi et la souffrance du fils à ce père longtemps inconnu, auteur du mensonge dans lequel vécut l'enfant jusqu'à son départ à la guerre. Mais on peut aussi y lire la parole «prolongée» par le fils de Louis Andrieux : on sait que celui-ci, qui n'avait pas confiance en ses fils légitimes, confia à Aragon le soin de parler en son nom pour écarter toute récupération religieuse sur son lit de mort. La transposition autobiographique concernant le père d'Aragon est transparente, aussi bien dans l'évocation de son «péché » que dans celle de sa faiblesse à l'article de la mort sous la pression de la «visiteuse de la dernière minute», qui obtint de l'agnostique une «conversion in extremis $^{23}$ ». Les rapports complexes d'Aragon avec le père ont été étudiés remarquablement par Roselyne CollinetWaller ${ }^{24}$. Contemporain du Fou d'Elsa, Le Mentir-vrai, écrit en 1964, aborde explicitement le «secret» de la filiation d'Aragon, et la façon dont elle lui fut révélée en 1918 quand il partit pour le front. Il y fait même référence au Fou d'Elsa : «dans Le Fou d'Elsa l'enfant Boabdil peut-être me ressemble qui dit: "Je n' aimais pas mon père" 25 ".

Au-delà de cette intrusion de l'histoire personnelle de l'auteur dans le poème, celui-ci reste avant tout une déclaration résolue d'athéisme et comme le message dernier d'Aragon. Cette fin prolonge certaines discussions sur le mal entre le roi Boabdil et le Medjnoûn, qui suggérait au roi d' «abandonner le prédicat de Dieu» (p. 297), et elle écarte l'ambiguïté possible d'une interprétation mystique de la relation amoureuse. Le poème se place sur un autre plan que «Le vrai zadjal...».

20. Jacques Berque, «Zadjal pour une Grenade possible», L'Arc, n 53 «Aragon », Aix-en-Provence, 1973, p. 66.

21. Aragon dit dans le «Lexique» du Fou d'Elsa que «Simorgh est une allégorie de Dieu ».

22. «[Cet ange] se tint en majesté, alors qu'il était à l'horizon supérieur. Puis il s'approcha et demeura suspendu et fut à deux arcs ou moins » (Coran, sourate «L'étoile», trad. Régis Blachère, Paris, Maisonneuve et Larose, 1956).

23. Voir le récit de l'agonie de Mercadier dans Les Voyageurs de l'impériale, et les confidences tardives sur les derniers jours de Louis Andrieux dans Henri Matisse, roman II, Livre Club Diderot : chapitre «Que 1'un fût de la chapelle», texte écrit par Aragon en 1969: «Ce père illégitime que j'ai eu», p. 213 à 217 : «il entendait avoir en moi un témoin de ce que tel jour, à telle date, ayant encore sa pleine lucidité d'esprit, n'ayant en rien changé les convictions de toute sa vie, il m'avait dit pour que je le répète, si c'était nécessaire, qu'il ne croyait pas en Dieu [...] Et ce qu'il me demandait, si jamais on avait obtenu de lui un reniement de lui-même, c'était d'élever la voix et de parler en son nom.»

24. Roselyne Collinet-Waller, Aragon et le père, romans, Presses universitaires de Strasbourg, 2001. Voir notamment p. 103 et 124-125.

25. $O P C$, p. 1333 
La place finale retenue pour «Ô impie» conclut certes l'œuvre dans le prolongement de certains débats théologiques, dont Wolfgang Babilas a précisé les termes ${ }^{26}$.

\section{Conclusion}

Les trois poèmes des «Apocryphes» sont donc, par la forme et la prosodie, très différents et issus d'un processus génétique complexe. Le premier, écrit en vers de seize syllabes, dit la lutte incessante en nous, dans notre corps, de la vie et de la mort, le battement de tambour du cœur et du sang dans les veines, et cette contradiction de tous les instants qui s'appelle vivre :

Si je parle je me détruis et ce que je dis est ma cendre

Et la ténèbre devant moi c'est ma lumière qui l'engendre.

Le dernier vers toutefois exprime le vœu que de cette contradiction naisse l'harmonie.

«Le vrai zadjal d'en mourir», en quatrains d'octosyllabes, commence comme une déclaration d'amour murmurée à la première personne : elle s'élargit, répétée, par le passage à la troisième personne pour devenir progressivement une proclamation d'amour fou.

Le troisième poème, commencé par la méditation du mourant sur ce qu'il a fait de sa vie, devient très vite l'interpellation véhémente, iconoclaste et railleuse de ce Dieu qui prétend être reconnu comme Créateur. Vers la fin, elle fait place à l'acceptation stoïcienne de l'anéantissement dans la fusion avec la nature27. Là aussi l'écriture suit de près ces mouvements : le début et la fin du poème sont écrits dans un temps du passé qui décrit de loin l'homme méditant, tandis que le corps principal du poème est au présent et à la première personne.

Ces trois poèmes se prêtent à plusieurs types d'analyse génétique. «Et si beau que me fût le jour » offre la situation «classique» d'un texte dont on peut suivre la genèse à travers ses remaniements successifs. Déplacé d'un endroit à l'autre de l'œuvre, il nous apporte aussi une indication précieuse sur le travail d' "ordonnancement» auquel se livre L'auteur. «Le vrai zadjal d'en mourir» semble au contraire avoir été écrit d'un seul jet pour répondre à une attente, combler un vide dans un ensemble dont la dynamique est jusqu' au dernier moment en gestation. En ce qui concerne «Ô Impie», pour lequel nous n'avons que très peu de variantes, c'est l'intertextualité avec le Coran qui apporte un éclairage sur le premier paragraphe et sur le dernier vers. Et c'est l'intertextualité, au sein même du Fou d'Elsa, avec le chapitre «Débat de l'avenir» qui en fournit le contrepoint.

On peut s'interroger sur le rapport des «Apocryphes» à l'ensemble du poème. Le Fou d'Elsa se nourrit d'une réflexion inquiète sur l'histoire des hommes et des civilisations, sur les espérances, les conflits, les tragédies dont elle est pleine. L'attente et l'angoisse de l'avenir habitent le présent, tous les présents; et le Medjnoûn dans sa folie traverse les temps de l'Histoire.

Avec la mort du Medjnoûn nous entrons dans une autre dimension.

C'est ici que dans moi s'arrête cette histoire et s'efface Grenade et je ne suis que moi c'est ici qu'à la fin je mesure ma force ou du moins ma faiblesse [...] et le jour qui s'achève et la pierre à mes pieds...28

Le tohu-bohu de l'histoire fait place tout d'un coup à ce temps suspendu, cet intemporel dans lequel se déploient les trois poèmes.

Qu'il s'agisse du roulement de tambour de la vie et de la mort, du vrai zadjal qui sublime l'amour dans la mort, ou du face à face de l'homme avec Dieu, c'est toujours un homme seul, «seul absolument seul inutilement nu29 ${ }^{2}$ » qui parle, ramené à sa nudité comme à son humanité essentielle. Est-il alors dans la continuité de tout ce qui a précédé ou au seuil d'une méditation nouvelle? C'est la question qu'on peut se poser.

26. Wolfgang Babilas, Études sur Louis Aragon, «Dieu dans Le Fou d'Elsa», Münster, Nodus Publikationen, 2002.

27. On peut songer ici à ce qu'Aragon écrit de Matisse qui «parlait parfois de se faire incinérer, dans un esprit panthéiste, aimant à penser que ce qui resterait de lui, si on dispersait ses cendres, se trouverait retourner à la nature, emporté dans le ciel, ou retombant sur la terre pour se mêler aux champs, aux forêts, à la mer, aux sables des plages » (Henri Matisse roman II, p. 226-227).

28. Édition originale, p. 401 / OPC II, p. 881.

29. «La grotte», XXII, édition originale, p. 390 / OPC II, p. 872. 
SuZANNE RAVIS-FrANÇON a soutenu en 1991 une thèse d'État sur le temps dans l'œuvre romanesque d'Aragon et enseigné de 1968 à 1999 la littérature française contemporaine à l'Université de Provence. Elle a dirigé une dizaine de thèses sur l'œuvre d'Aragon, organisé plusieurs colloques et animé pendant des années le groupe de recherche ERITA. Elle a coordonné le colloque et le volume collectif Le Rêve de Grenade. Aragon et Le Fou d'Elsa (Publications de l'Université de Provence, 1996) et publié, à partir du manuscrit, de nombreux articles sur Le Fou d'Elsa.

\section{Réécriture et recomposition dans le manuscrit du Fou d'Elsa}

Par leur place à la fin de l'ouvrage, et par leur titre énigmatique, les «Apocryphes des derniers jours» appellent réflexion et analyse. Ils sont en effet le fruit d'un long travail d'écriture et de composition : déplacement de textes, écriture de dernière heure, presque «sur le marbre» pour l'un des poèmes, intertextualité et remaniements importants. À partir du manuscrit, l'analyse génétique et/ou intertextuelle privilégie deux types d'approche distincts, mais liés l'un à l'autre : la composition «en mouvement» du sous-ensemble constitué par ces trois textes et l'observation détaillée des états successifs du premier poème des «Apocryphes».

Given their place at the end of the book and their enigmatic title, the "Apocrypha of the last days" call for reflection and analysis. They are indeed the fruit of a considerable amount of writing and composition: moving texts, last-minute writing, at the printing press for one of the poems, rearrangements and other important reworkings. For the manuscript, the genealogical and/or intertextual analysis privileges two distinct but interrelated types of approach: the examination of the rearrangements of these three texts, and detailed observation of the successive states of the first poem of the "Apocrypha".

Durch ihren Platz am Ende des Buches und ihren rätselhaften Titel rufen die „Apocryphes des derniers jours“ zum Nachdenken und zur Analyse auf. Sie sind in der Tat das Ergebnis einer langen Schreib- und Kompositionsarbeit: Textverschiebungen, Schreiben in letzter Minute, gar kurz vor dem Druck für eines der Gedichte, Intertextualität und wichtige Überarbeitungen. Ausgehend vom Manuskript favorisiert die genetische und/oder intertextuelle Analyse zwei unterschiedliche, aber verwandte Ansätze: den Fokus auf die verschiedenen Momente der Zusammensetzung der Untermenge, die diese drei Texte bilden, und die detaillierte Beobachtung der aufeinanderfolgenden Zustände des ersten Gedichts der „Apocryphes“.
Por su ubicación al final de la obra y por su título enigmático, los "Apócrifos de los últimos días" requieren reflexión y análisis. Son, en efecto, el fruto de un largo trabajo de escritura y composición: desplazamiento de textos, escritura de último minuto -casi en prensa, por lo que se refiere a uno de los poemas-, intertextualidad y modificaciones importantes. A partir del manuscrito, el análisis genético y/o intertextual privilegia dos tipos de enfoques distintos, pero ligados uno al otro: la composición "en movimiento" del subconjunto constituido por estos tres textos y la observación detallada de los estadios sucesivos del primer poema de los "Apócrifos".

Por estarem no final da publicação e pelo título enigmático, os «Apocryphes des derniers jours»/ / Apócrifos dos últimos dias» chamam à reflexão e à análise. Com efeito, eles são o fruto de um longo trabalho de escrita e de composição: deslocamento de textos, escrita de última hora e, no caso de um dos poemas, quase à beira da impressão, intertextualidade e remodelações importantes. A partir do manuscrito, a análise genética e/ou intertextual privilegia dois tipos de abordagem distintos, mas ligados um ao outro a composição « em movimento » do sub-conjunto constituído por esses três textos e a observação detalhada dos estados sucessivos do primeiro poema dos «Apócrifos».

Posti alla fine dell'opera e dotati di un titolo enigmatico, gli "Apocrifi degli ultimi giorni" invitano alla riflessione e all' analisi. Essi sono il frutto di un lungo lavoro di scrittura e di composizione: spostamento di testi, scrittura dell'ultimo momento (un poema viene scritto quasi sul torchio di stampa), intertestualità e rimaneggiamenti importanti. L'analisi genetica e/o intertestuale privilegia due tipi di approccio del manoscritto, differenti ma legati tra loro: la composizione "in movimento" del sottoinsieme costituito da questi tre testi e l'osservazione dettagliata delle versioni successive del primo poema degli "Apocrifi". 